\title{
Linear parameter-varying modelling and control of an offshore wind turbine with constrained information
}

\author{
Tore Bakka ${ }^{1}$, Hamid Reza Karimi ${ }^{1}$, Søren Christiansen ${ }^{2}$ \\ ${ }^{1}$ Department of Engineering, Faculty of Engineering and Science, University of Agder, N-4898 Grimstad, Norway \\ ${ }^{2}$ Department of Electronic Systems, Aalborg University, Aalborg, Denmark \\ E-mail: hamid.r.karimi@uia.no
}

Received on 28th May 2013

Revised on 12th August 2013

Accepted on 18th September 2013

\section{Nomenclature}

Throughout this paper, the notations $\mathcal{R}^{n}$ and $\mathcal{R}^{n \times m}$ denote, respectively, the $n$-dimensional Euclidean space and the set of all $n \times m$ real matrices. Superscript $T$ denotes matrix transposition and $I$ and 0 are the identity matrix and the zero matrix with compatible dimensions, respectively. The symbol $\otimes$ denotes Kronecker product of two matrices. The notation $P>0$ means that $P$ is real symmetric and positive definite and the symbol $*$ denotes the transpose elements in the symmetric positions, $\operatorname{diag}\{\cdots\}$ represents a block diagonal matrix and the operator $\operatorname{sym}(A)$ represents $A+A^{\mathrm{T}}$.

\section{Introduction}

In recent years, there has been an increasing interest in wind energy. According to The World Wind Energy Association, the worldwide wind energy reached a capacity of 254000 MW in June 2012 [1]. Although the majority of the installed capacity is on land, many offshore parks have been built in recent years. Most of these parks have turbines that are either fixed to the soil or they stand on monopoles or other structures. Some of the major offshore wind farms in Europe are located in the United Kingdom (UK) and Denmark (DK), to name a few; Greater Gabbard (UK), Whalney (UK), Sheringham Shoal (UK), Horns Rev (DK) and Rødsand (DK). The turbines in these farms are installed in shallow waters, typical depths ranging from 10 to $30 \mathrm{~m}$ [2]. For many countries such as Spain, United States, Japan, Korea and Norway it would be beneficial to also be able to install wind turbines in deeper waters, in depths up to several hundred metres. The existing offshore fixed-bottom wind turbines are not suited for such deep water. Hywind [3] is one example of a floating wind turbine solution. This turbine was installed back in 2009 and is still in operation. It is located in the north sea, right off the Norwegian west coast. This paper deals with a model that is based on Hywind, a sketch of the turbine can be seen in Fig. 1.

In order to keep wind turbines stable and in operation for a long as possible, it is important to control them in a suitable way. The region of operation for a typical wind turbine is often divided into four regions; see Fig. 2. In region I $\left(v<v_{\text {cut-in }}\right)$, the wind speed is lower than the cutin wind speed and no power can be produced. In region II $\left(v_{\text {cut-in }}<v<v_{\text {rated }}\right)$, the blade pitch is usually kept constant, while the generator torque is the controlling variable. In this region, the main objective is to maximise the power 


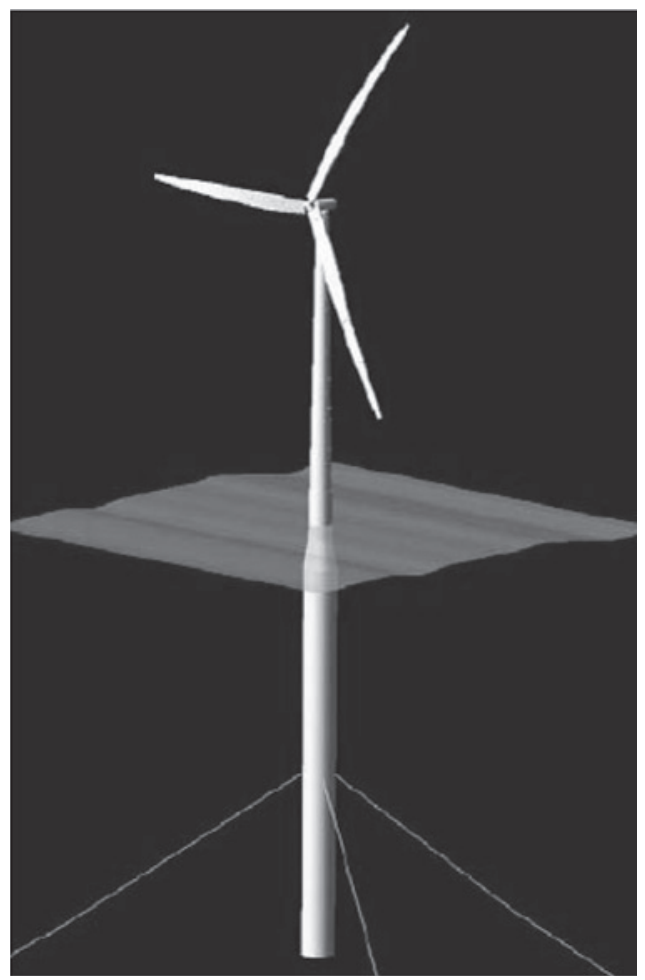

Fig. 1 Sketch of Hywind turbine [4]

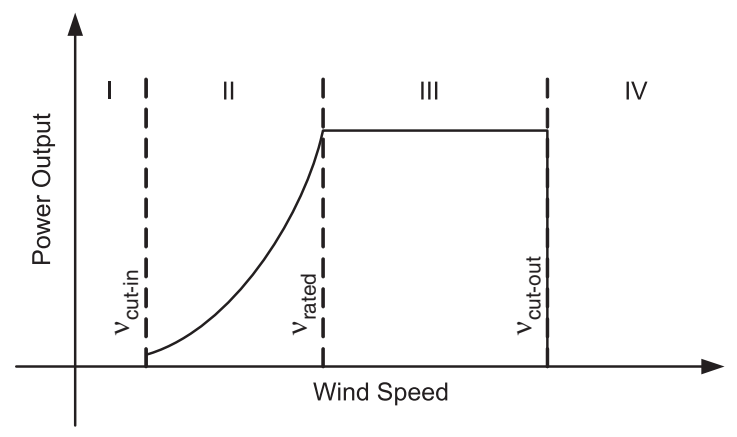

Fig. 2 Region of operation for a typical wind turbine

output. In region III $\left(v_{\text {rated }}<v<v_{\text {cut-out }}\right)$, the main concern is to keep the rated power and speed simultaneously. This is achieved by means of pitching the blades into or out of the wind, depending on the wind situation. In region IV $\left(v>v_{\text {cut-out }}\right)$, the wind speed is too high, and the turbine is shut down. This paper is focused on the above-rated wind speed scenario, that is, region III.

Advanced control techniques are often solved by formulating the problem in terms of linear matrix inequalities (LMIs) [5]. By formulating the problem in such a way, gives an opportunity to impose a special zero-non-zero structure on the LMI variables. This comes in very handy when dealing with constrained information systems. Linear parameter-varying (LPV) systems can also be handled within the LMI framework. Recently, linear controllers have been extensively used for power regulation through the control of blade pitch angle in wind turbine systems. However, the performance of these linear controllers is limited by the highly non-linear characteristics of the wind turbine. Advanced control is one research area where such improvement can be achieved. On the other hand, over the last three decades, considerable attention has been paid to robustness analysis and control of linear systems affected by structured real parameters, so-called LPV systems. An LPV system can be viewed as a non-linear system that is linearised along a trajectory determined by the parameter vector. Hence, the parameter vector of an LPV system corresponds to the operating points of the non-linear system. In the LPV framework, it is assumed that the parameter vector is measurable or non-measurable. In the latter case, the parameter vector can be estimated. In many industrial applications, such as flight control and process control, the operating point can indeed be determined from measurement, making the LPV approach viable. Interesting works where LPV control is used in relation to wind turbines can be found in references such as [6-8]. A nice collection of LPV related papers can be found in [9], in addition to [10-12]. Previous related works from the authors which are dealing with wind turbine control can be found in $[13,14]$.

State-feedback is widely used in control applications, but in practice full state measurements are rarely possible. A more practical approach is output-feedback. However, the output gain matrix is not computed as easy as in the statefeedback case, where a simple change of variables converts a non-convex problem into a convex problem. In the outputfeedback case, the gain matrix is not directly isolated from the other LMI variables. In [15-17], the authors propose an explicit solution for the gain matrix and in [18] the authors develop a more effective and efficient method for computational feasibility issues of static output-feedback controller gains. With the method found in [18], it is possible to impose zero-non-zero constraints on the LMI variables. Other methods to make the system more tolerant to failures have been investigated in [19-21].

This paper is dealing with an offshore floating turbine, now the control design becomes even more important. The wrong control design could easily force the turbine to be shut down. Nowadays, modern wind turbines are getting bigger and bigger and are often located in harsh environments, such as offshore conditions. This leads to larger loads and there is always the possibility of sensor failure. Since immediate maintenance for these turbines are not possible, a way to keep them in operation according to prescribed performance measures would be beneficial. This paper tries to include four concrete issues in the controller design. (1) Limit oscillations in drivetrain and platform. (2) Keep generator speed at its rated value. (3) Limit influence on the control loop in case of sensor failure. (4) The controller is designed within the LPV framework. In this relation an individual pitch with constrained gain control scheme is suggested. The ability to impose special structure on the gain matrix come in handy if for example; there are some sensors in the feedback loop that are especially prone to failure. In this way, one can isolate the faulty measurement to only impact one of the pitch actuators, instead of all three. A diagonal structure for the gain matrix will be used, in this way, none of the pitch actuator signals will directly interfere with each other. In conventional control, in the worst case, the turbine might have to shut down because a sensor is malfunctioning. With the control proposed in this paper, the turbine can continue operating properly in the event of a failure. Even though, the failure should be fixed as soon as possible. The main contribution of this paper is to design an LPV controller under a constrained gain matrix for a floating wind turbine system.

This paper is organised as follows. In Section 2, an LPV model of the wind turbine system is proposed. The controller design is discussed in detail in Section 3, where it will be shown how it is possible to obtain a diagonal structure 
for the gain matrix. In Section 4, the simulation results are presented. Conclusions and suggestions for future work are described in Section 5.

\section{Model description}

The model used in this paper is obtained from the software fatigue, aerodynamic, structural and turbulence (FAST) [22], which is a fully non-linear wind turbine simulation software. The OC3-Hywind model is a $5 \mathrm{MW}$ offshore floating turbine [23], its main specifications are listed in Table 1. The turbine model is an up scaled version of Statoils 2.3 MW Hywind Demonstration turbine.

The non-linear model obtained from FAST, is linearised around several operating conditions. All conditions are above-rated wind speed, which indicates that the controller objectives are the same for each point. The objectives should be achieved by means of individual pitching of the blades. The non-linear model consists of 24 degrees of freedom (DOFs). This is all the available DOFs in FAST, if one desires, it is easy to switch the different DOFs on and off. To be able to do this is convenient if just a simple model is necessary, which is needed for the linearised model. The DOFs for the linear model are selected while keeping the controller objectives in mind, these include; platform pitch, generator and rotor dynamics. Pitch actuators will be added later in Section 2. As a consequence, three additional DOFs will be added to the linear model. In total, there are nine DOFs with 18 states, two for each DOF (position and velocity). With the linear model, most of the dynamics that are relevant for the controller objectives should be reflected. There are three measured signals in the feedback loop; generator speed, rotor speed and platform pitch angle. By using these three signals in the feedback loop, the three blade pitch angles can be calculated. A set of nine linearisation points, ranging from 14 to $22 \mathrm{~m} / \mathrm{s}$, are obtained. The linearised models obtained from FAST are on the following standard state-space form

$$
\begin{aligned}
& \dot{x}=A_{i} x+B_{i} u, \quad i=1, \ldots, 9 \\
& y=C x
\end{aligned}
$$

where the state-space matrices $A_{i}, B_{i}$ and $C$ are of dimensions $\mathcal{R}^{n \times n}, \mathcal{R}^{n \times m}$ and $\mathcal{R}^{q \times n}$, respectively, where $n, m$ and $q$ are 6, 3 and 3 , respectively. From system (1) it is seen that $A_{i}$ and $B_{i}$ vary, depending on the operating point, that is, wind speed. While the $C$ matrix is constant since it only handles the measurements. The system can now be formulated on a generalised form in (2).

$$
\begin{aligned}
& \dot{x}=A_{i} x+B_{i} u+B_{w} \omega, \quad i=1, \ldots, 9 \\
& z=C_{z} x+D_{z} u \\
& y=C x
\end{aligned}
$$

Table 1 Main specifications of NREL's OC3 turbine

\begin{tabular}{ll}
\hline Rated power & $5 \mathrm{MW}$ \\
\hline Rated wind speed & $11.6 \mathrm{~m} / \mathrm{s}$ \\
Rated rotor speed & $12.1 \mathrm{RPM}$ \\
Rotor radius & $63 \mathrm{~m}$ \\
Hub hight & $90 \mathrm{~m}$ \\
\hline
\end{tabular}

where the additional term $B_{w}$ describes how the disturbance enters the system, $C_{z}$ and $D_{z}$ handle the performance measures, that is, the controller objectives.

So far, one state-space system for each operating point is obtained. In order to utilise the suggested control technique a continuous model of the set of state-space systems is needed. To achieve this, least mean square method is used to obtain a polynomial of the first order. The method fits a polynomial to the data in the matrices and calculates the polynomial coefficients that gives the least square error between the suggested polynomial and the data points. A continuous affine model is thus obtained. The two parameter-dependent matrices are defined in the following affine way

$$
\begin{aligned}
& A(\sigma)=A_{a}+\sigma A_{b} \\
& B(\sigma)=B_{a}+\sigma B_{b}
\end{aligned}
$$

where the scalar parameter $\sigma$ satisfies $\underline{\sigma} \leq \sigma(t) \leq \bar{\sigma}$ and $\rho \leq \dot{\sigma}(t) \leq \bar{\rho}$, that is, $\sigma \in\left[\begin{array}{ll}\underline{\sigma} & \bar{\sigma}\end{array}\right]$ and $\rho \in\left[\begin{array}{ll}\rho & \bar{\rho}\end{array}\right]$.

From (3) it is seen that the state-space system is now parameter-dependent on $\sigma$, which is the wind speed. The wind speed will later in the paper be estimated for this purpose.

As FAST does not include any pitch actuators, these are added to the parameter-dependent state-space system. One pitch actuator system is considered for each blade. As seen in the combined system (5), all the parameter dependency is limited to the $A$ matrix.

$$
\begin{aligned}
\dot{\tilde{x}} & =\tilde{A}(\sigma) \tilde{x}+\tilde{B} u+\tilde{B}_{w} \omega \\
\tilde{z} & =\tilde{C}_{z} \tilde{x}+D_{z} u \\
\tilde{y} & =\tilde{C} \tilde{x}
\end{aligned}
$$

where the new augmented state $\tilde{x}=\left[\begin{array}{ll}x_{p}^{\mathrm{T}} & x^{\mathrm{T}}\end{array}\right]^{\mathrm{T}}$ has the following corresponding state-space matrices:

$$
\begin{aligned}
\tilde{A}(\sigma) & =\underbrace{\left[\begin{array}{cc}
I_{3} \otimes A_{p} & 0 \\
B_{a} \otimes C_{p} & A_{a}
\end{array}\right]}_{\tilde{A}_{a}}+\sigma \underbrace{\left[\begin{array}{cc}
0 & 0 \\
B_{b} \otimes C_{p} & A_{b}
\end{array}\right]}_{\tilde{A}_{b}} \\
\tilde{B} & =\left[\begin{array}{c}
I_{3} \otimes B_{p} \\
0
\end{array}\right] \\
\tilde{C} & =\left[\begin{array}{ll}
0 & C
\end{array}\right]
\end{aligned}
$$

where $A_{p}, B_{p}$ and $C_{p}$ are the blade pitch actuator statespace matrices (see the appendix for matrix values), $\tilde{B}_{w}$ is defined in Section 4, $\tilde{A}_{a}$ and $\tilde{A}_{b}$ are the new augmented matrices of (3). The updated dimensions are now $n=12$ and $m=q=3$.

\section{Control synthesis}

This section mainly deals with the controller design process and briefly discusses the extended Kalman filter (EKF). The main objective is to design an output-feedback LPV controller which is able to handle structure constraints on the output-feedback gain matrix. The LPV controller depends on the wind speed parameter, which is estimated by the EKF. Fig. 3 shows a block diagram, the block diagram describes the basics of the closed-loop system. In the first feedback loop, three values $\left(y_{1}\right)$ are used to estimate the effective wind speed $(\sigma)$. In the second feedback loop, three sensor 


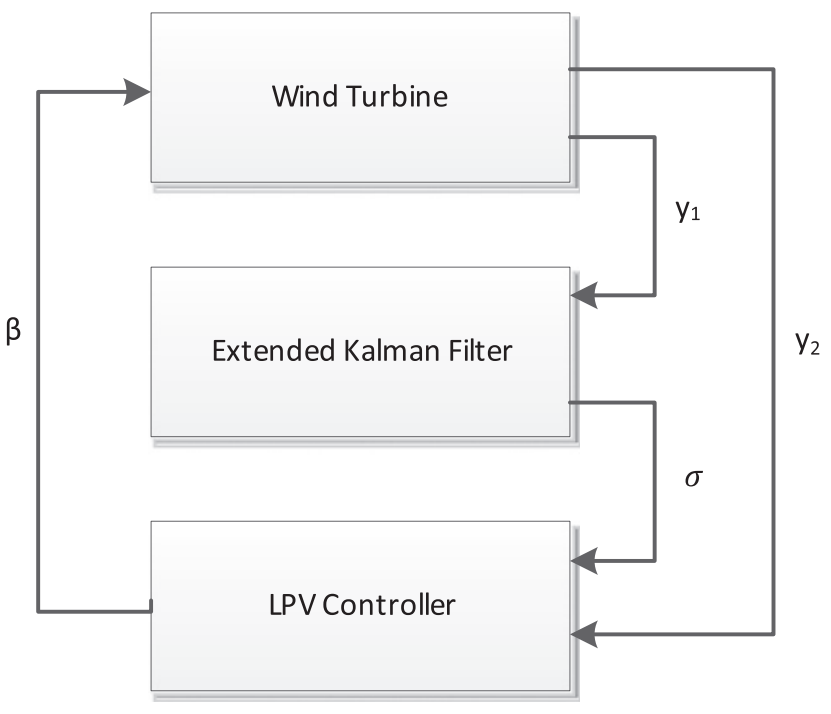

Fig. 3 Block diagram of closed-loop system

values $\left(y_{2}\right)$ are used to calculate the three blade pitch angles $(\beta)$. These output values correspond to (5). As discussed in Section 2 , this means the feedback gain is a $3 \times 3$ matrix. This section considers calculation of an output-feedback gain matrix with a diagonal structure. In this way, not all the available information will be used to calculate each of the blade pitch angles and none of the three control signals will directly interfere with each other. That is, should a failure happen to for instance sensor one, then this will not directly influence control signal two or three.

\subsection{Extented Kalman filter}

It is possible to estimate the effective wind speed based on measurements of rotor speed, blade pitch angle and generator torque. The effective wind speed represents the average wind field over the rotor disc, that is, what is experienced by the blades. An EKF is used based on a simple model of the drivetrain and a turbulence model, the output from the EKF is the effective wind speed. For the actual development of the filter, readers are referred to [24].

The drivetrain and wind model of the wind turbine is modelled as first-order systems with no losses.

$$
\begin{aligned}
J_{\mathrm{eq}} \dot{\Omega}_{D T} & =T_{a}-T_{g} \\
\dot{v}_{t} & =-\frac{\pi v_{m}}{2 L}+n_{1} \\
\dot{v}_{m} & =n_{2} \\
\sigma & =v=v_{m}+v_{t}
\end{aligned}
$$

where $J_{\text {eq }}=J_{r}+n_{g}^{2} J_{g}, T_{g}=T_{e} n_{g}, v_{t}$ is turbulence, $v_{m}$ is the mean wind speed and $L$ is the turbulence length scale parameter. The wind model is driven by Gaussian white noise, entering the model by $n_{1}$ and $n_{2}$. This model is non-linear because of the non-linear relationship between wind speed and aerodynamic torque. In order to estimate the states, the time update uses information about the model dynamics and the model uncertainties.

$$
\begin{aligned}
& \hat{x}_{k}^{-}=A_{e k f} \hat{x}_{k-1}+B_{e k f} u_{k-1} \\
& P_{k}^{-}=A_{e k f} P_{k-1} A_{e k f}^{\mathrm{T}}+Q
\end{aligned}
$$

where matrices $\left(A_{e k f}, B_{\text {ekf }}, C_{\text {elf }}\right)$ are state-space matrices of a linearised version of (10)-(11), $Q$ is the incremental process noise covariance and $P_{k}$ is the state estimate error covariance. The measurement update uses information about the model outputs and measurement noise.

$$
\begin{aligned}
K_{k} & =P_{k}^{-} C_{e k f}^{\mathrm{T}}\left(C_{e k f} P_{k}^{-} C_{e k f}^{\mathrm{T}}+R\right) \\
\hat{x}_{k} & =\hat{x}_{k}^{-}+K_{k}\left(z_{k}-C_{e k f} \hat{x}_{k}^{-}\right) \\
P_{k} & =\left(I-K_{k} C_{e k f}\right) P_{k}^{-}
\end{aligned}
$$

where $R$ is measurement noise covariance, $K_{k}$ is the Kalman gain and $z_{k}$ is the measurements.

\subsection{Controller design}

For obtaining the results of $H_{\infty}$ controller synthesis, the wind speed parameter in (5) needs to be estimated according to subsection $A$.

Definition 1: The $H_{\infty}$ performance measure of system (5) is defined as $J_{\infty}=\int_{0}^{\infty}\left(z^{\mathrm{T}} z-\gamma^{2} \omega^{\mathrm{T}} \omega\right) \mathrm{d} t$, where the positive scalar $\gamma$ is given.

As described in [5], the $H_{\infty}$ constraint $J_{\infty}<0$ for the linear time-invariant (LTI) system (5) with state-feedback is formulated as follows

$$
\begin{aligned}
\left(\begin{array}{cc}
\operatorname{sym}(\tilde{A} X+\tilde{B} Y)+\gamma^{-2} \tilde{B_{w}}{\tilde{B_{w}}}^{\mathrm{T}} & \left(C_{z} X+D_{z} Y\right)^{\mathrm{T}} \\
* & -I
\end{array}\right)<0 \\
X>0
\end{aligned}
$$

Remark 1: The number of variables to be determined in LMIs (19)-(20) are: $n \times(1+n) / 2+m \times n+1$.

In the state-feedback case, the gain matrix is calculated from $K=Y X^{-1}$. In the output-feedback case, the gain matrix factors as the product $\tilde{K}=K \tilde{C}$, where $\tilde{C}$ is given from (9). In order to obtain the output-feedback gain, a solution to (19)-(20) needs to be found such that the product $Y X^{-1}$ factors as

$$
K \tilde{C}=Y X^{-1}
$$

In a recent work by [18], a procedure that handles this problem in a very systematic way is developed. Another benefit with this approach is that it is possible to impose structural constraints on the gain matrix. The following change of variables are suggested

$$
\begin{aligned}
X & =Q X_{Q} Q^{\mathrm{T}}+R X_{R} R^{\mathrm{T}} \\
Y & =Y_{R} R^{\mathrm{T}}
\end{aligned}
$$

where $X_{Q}(\sigma)$ and $X_{R}(\sigma)$ are $\mathcal{R}^{(n-q) \times(n-q)}$ and $\mathcal{R}^{(q \times q)}$ symmetric matrices, respectively, and $Y_{R}(\sigma)$ is a $\mathcal{R}^{(m \times q)}$ matrix. The $Q$ matrix is the nullspace of $C$, and $R$ can be calculated from the following expression

$$
R=\tilde{C}^{\mathrm{T}}\left(\tilde{C} \tilde{C}^{\mathrm{T}}\right)^{-1}+Q L
$$

where $L$ is an arbitrary matrix with dimensions $\mathcal{R}^{(n-q) \times q}$. One of the main contributions in this paper is to force the Lyapunov matrices to be parameter-dependent. The Lyapunov 
matrices (25) are now portioned in the same affine way as the matrices $A(\sigma)$ and $B(\sigma)$ in (3).

$$
\begin{aligned}
X_{Q}(\sigma) & =X_{Q 0}+\sigma X_{Q 1} \\
X_{R}(\sigma) & =X_{R 0}+\sigma X_{R 1} \\
Y_{R}(\sigma) & =Y_{R 0}+\sigma Y_{R 1}
\end{aligned}
$$

By changing $v=\gamma^{-2}$ in the LMIs (19)-(20), the problem becomes convex and by maximising $v$ it is possible to find the Lyapunov matrices in (25). In order to obtain the diagonal structure for the output-feedback gain the LMI variables $X_{R}(\sigma)$ and $Y_{R}(\sigma)$ also have to have a diagonal structure. Choosing the Lyapunov matrices as described, the outputfeedback gain can be calculated from $K(\sigma)=Y_{R}(\sigma) X_{R}^{-1}(\sigma)$. The interested reader can read the proofs in [18]. In order to guarantee the controller stability and performance within the bounds of the scheduling parameter, some additional LMIs are required. The system is depending on one parameter and as indicated earlier this parameter has an upper and a lower bound, both on the parameter itself and on the derivative. One LMI is needed to check each vertex, that is, this gives an addition of $2^{i}$ LMIs, where $i$ is the number of vertices. As a consequence of the parameter dependency, at the upper right position in the $H_{\infty}$ constraint (19), the expression is quadratic in $\sigma$. By imposing the definiteness of the terms involving $\sigma^{2}$, that is

$$
\operatorname{sym}\left(\tilde{A}_{b} Q X_{Q 1} Q^{\mathrm{T}}\right)+\operatorname{sym}\left(\tilde{A}_{b} R X_{R 1} R^{\mathrm{T}}\right) \geq 0
$$

the quadratic function of $\sigma$ is convex.

The total set of LMIs needed to solve the LPV constrained static output-feedback problem is represented in (27)-(30) where $i$ is the number of vertices. The derivative term of the Lyapunov function is found to be

$$
\frac{\partial X}{\partial \sigma}=Q X_{Q 1} Q^{\mathrm{T}}+R X_{R 1} R^{\mathrm{T}}
$$

From the optimisation the matrices $X_{Q 0}, X_{Q 1}, X_{R 0}, X_{R 1}, Y_{R 0}$ and $Y_{R 1}$ are obtained. At each time step during the simulation, a new value for $\tilde{A}(\sigma), X_{R}(\sigma)$ and $Y_{R}(\sigma)$ are calculated. The controller gain matrix $K(\sigma)$ is calculated from the expression $K(\sigma)=Y_{R}(\sigma) X_{R}^{-1}(\sigma)$. In this way, the outputfeedback controller will change depending on $\sigma$.

Remark 2: The number of variables to be determined in LMIs (27)-(30) are: $(n-q) \times(1+(n-q))+2 q+2 m+$ 1 . It is noted that when the structure constraints are imposed on the control problem, the number of variables are actually lower than in the standard static feedback case (Remark 1).

\section{Simulation results}

In this section, the different simulation results will be discussed and presented. The controllers are tested on the fully non-linear system, where all the available DOFs are enabled. The input to the system is a turbulent wind profile with an average wind speed of $18 \mathrm{~m} / \mathrm{s}$ with a turbulence intensity of $6 \%$ with corresponding waves. The wind profile is obtained from Turbsim [25], and is emulating a one year extreme wind speed condition. In the simulation example the plots concerning the two controllers designed in this paper experiences a fault after $500 \mathrm{~s}$. This is not the case for the baseline controller, where no fault occurs. The gain scheduled PI controller is not designed to handle such an event. The fault causes sensor three to stop working, that is, blade three has stopped moving see Fig. 8. The system is made in such a way that the actuator which controls blade number three keeps its last value prior the fault. In this way, the value does not drop to zero. Time series of $1000 \mathrm{~s}$ are obtained, but the first $400 \mathrm{~s}$ are removed because of transient behaviour. Each of the figures show simulations done with three different controllers. The blue curve shows simulation done with the constant constrained gain controller. The red curve is for the LPV constrained gain controller and the green curve is FASTs baseline controller. The baseline controller is intended as a reference plot and comes with the FAST package.

Fig. 4 shows the estimated states $\left(\hat{x}_{k}\right)$ and the Kalman gain values $\left(K_{k}\right)$. The plot also includes the simulated rotor speed (green) to compare with the estimated value (blue). As seen from the plot, the agreement is quite good.

As mentioned in Section 2, the controller objectives are to mitigate oscillations on critical parts of the system, to damp any movement which potentially can make the system unstable, such as platform pitch movement, and keep the overall system stable. These objectives are given as the performance measure $z$, as in (2) with the following matrices

$$
\begin{aligned}
& \tilde{B}_{w}=\left[1_{12 \times 1}\right]
\end{aligned}
$$

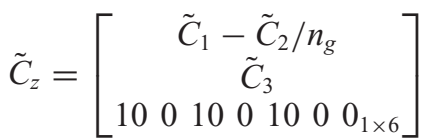

$$
\begin{aligned}
& D_{z}=\operatorname{diag}\left\{10^{3}, 10^{3}, 10^{3}\right\}
\end{aligned}
$$

$\tilde{B}_{w}$ is the disturbance matrix, and indicates which state that are disturbed. By giving the matrix only ones, the idea is to simulate a situation which is tougher than reality. $\tilde{C}_{i}$ indicates the ith row vector of the output matrix. The first row of $\tilde{C}_{z}$ handles the drivetrain oscillation, by subtracting the generator speed $\left(\tilde{C}_{2}\right)$ divided by the gearing ratio

$$
\begin{aligned}
& \left(\begin{array}{cc}
\operatorname{sym}\left(\tilde{A}\left(\sigma_{i}\right) X\left(\sigma_{i}\right)\right)+\operatorname{sym}\left(\tilde{B}\left(\sigma_{i}\right) Y\left(\sigma_{i}\right)+v \tilde{B}_{w} \tilde{B}_{w}^{\mathrm{T}} \pm \rho_{p} \frac{\partial X}{\partial \sigma}\right. & \left(C_{z} X\left(\sigma_{i}\right)+D_{z} Y\left(\sigma_{i}\right)\right)^{\mathrm{T}} \\
* & -I
\end{array}\right)<0, \quad i=p=1,2 \\
& \operatorname{sym}\left(\tilde{A}_{b} Q X_{Q 1} Q^{\mathrm{T}}\right)+\operatorname{sym}\left(\tilde{A}_{b} R X_{R 1} R^{\mathrm{T}}\right) \geq 0 \geq 0 \\
& \left(\begin{array}{cc}
X_{Q 0} & 0 \\
0 & X_{Q 1}
\end{array}\right)>0 \\
& \left(\begin{array}{cc}
X_{R 0} & 0 \\
0 & X_{R 1}
\end{array}\right)>0
\end{aligned}
$$



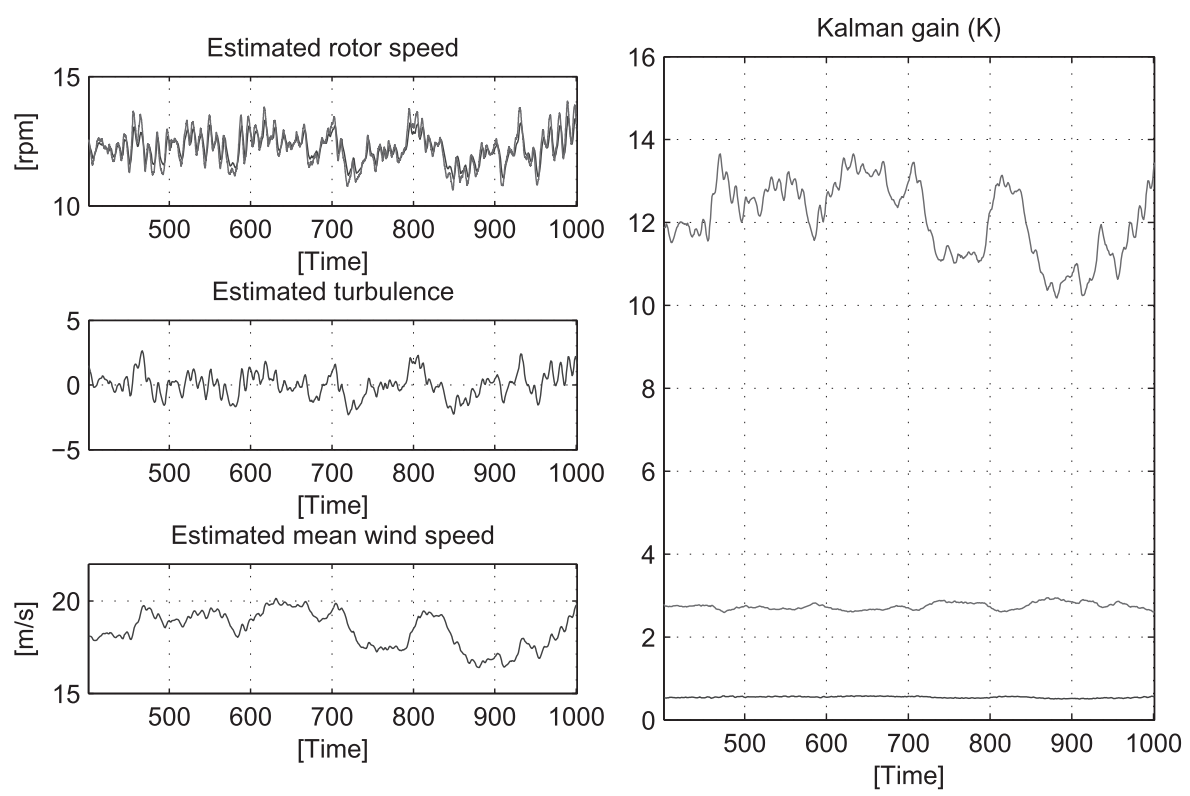

Fig. 4 Selected EKF parameters

$n_{\mathrm{g}}$ from the rotor speed $\left(\tilde{C}_{1}\right)$. The second and third row handle platform pitch - and blade pitch movement, respectively. Suitable results were found with a diagonal structure for the $D_{z}$ matrix. The same performance measure is used for both the blue and the red curve. The outcome from the LMI calculations are listed in the appendix, that is, the Lyapunov matrices used online to calculate the LPV constrained controller and the constant constrained controller. In order to check the drivetrain oscillations, the standard deviation for the speed difference between rotor speed and generator

Table 2 Normalised values for drivetrain oscillations

\begin{tabular}{ccc}
\hline Baseline & Constant controller & LPV controller \\
\hline 1 & 0.98 & 0.96 \\
\hline
\end{tabular}

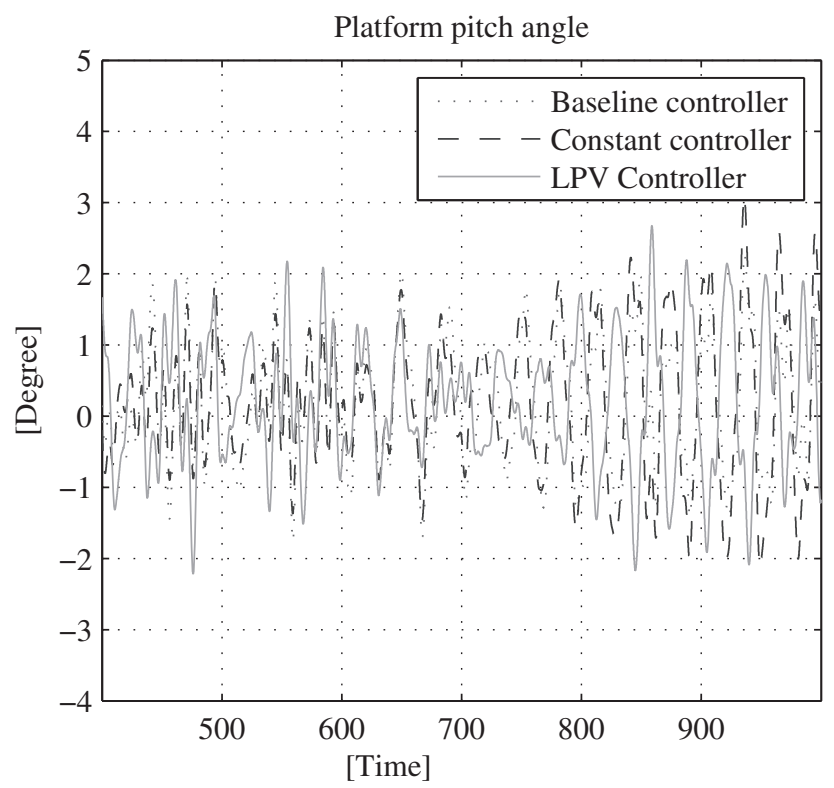

Fig. 5 Time series of platform pitch angle speed is calculated. The values are normalised. That is, the reference value is the baseline result, which is given value 1. For the remaining two controllers, a value below 1 indicates better and vice versa. Multiply by 100 to obtain the percentage.

From the Table 2 it is seen that the two controllers designed in this paper does damp the drivetrain oscillations slightly better than the baseline controller.

A time series plot of the generator speed with different controllers is presented in Fig. 6. It is seen that the constant constrained gain controller (dashed line) is slightly better than FASTs gain scheduled PI controller (dotted line). But the controller that gives the better responds is the LPV constrained gain controller (solid line). Several observations indicate this. First, it is operating more closely to the rated condition for the generator. Second, the power output does not fluctuate as much as the two others (Fig. 7). See also the mean and standard deviation plots in Figs. 9 and 10.

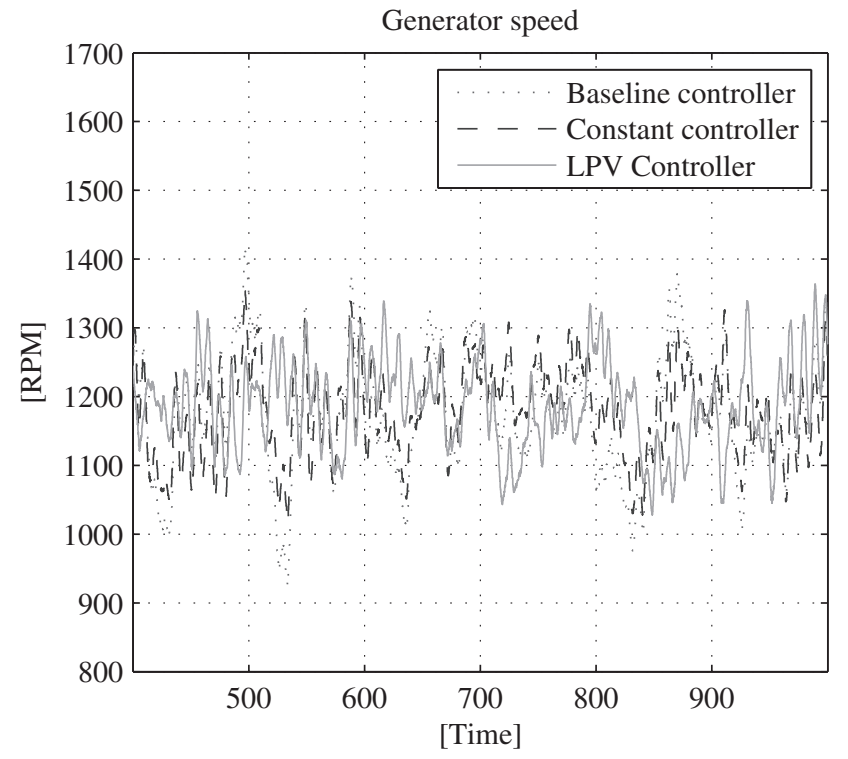

Fig. 6 Time series of generator speed 


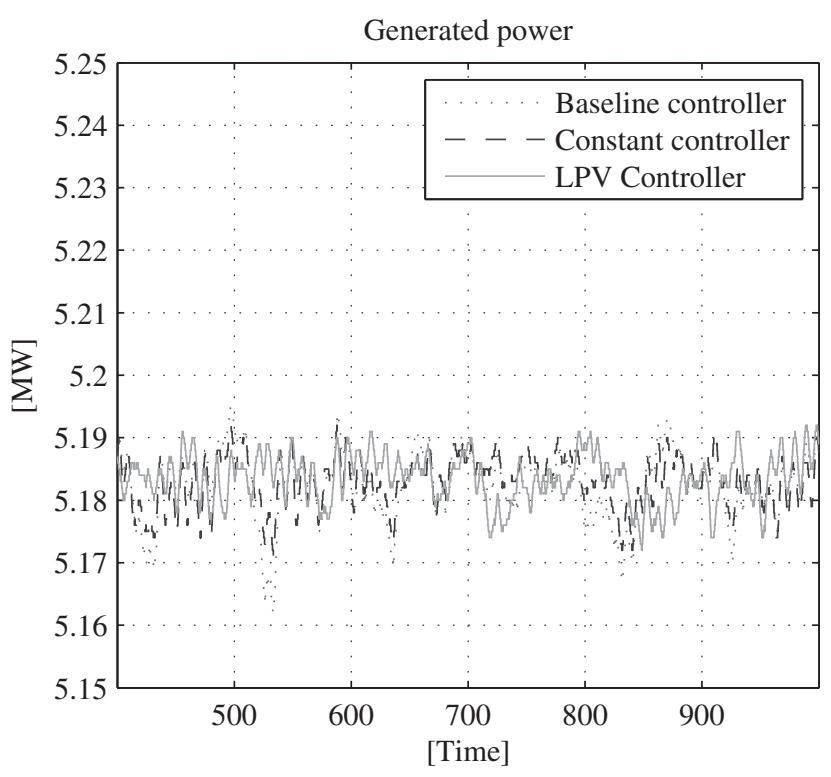

Fig. 7 Time series of generated power

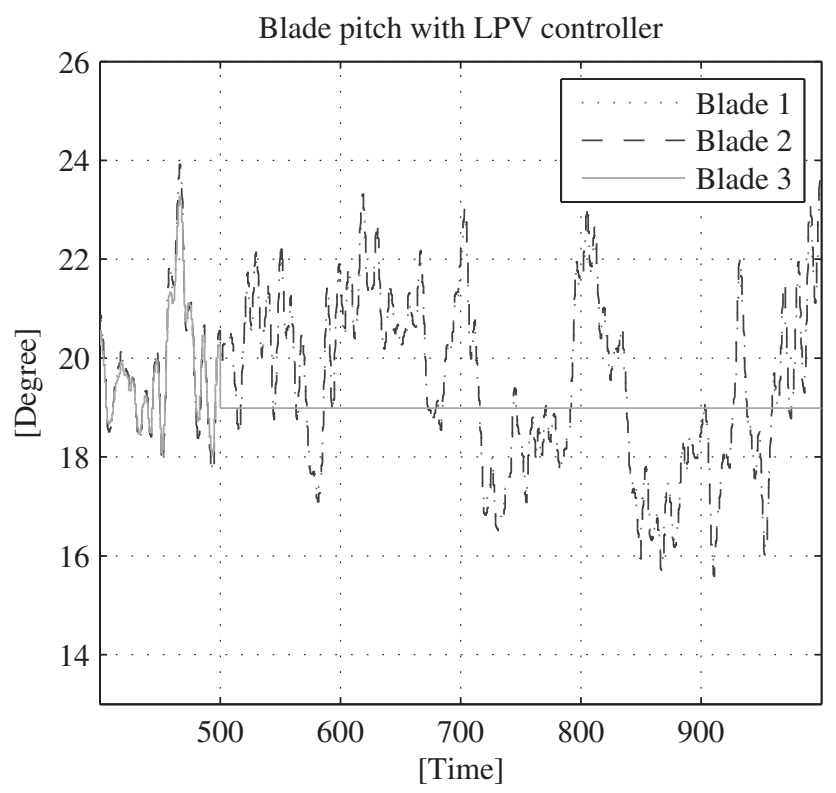

Fig. 8 Time series of blade pitch angles

With these performance measures and with the fault present in the system, is was not possible to dampen the platform pitch movement (Fig. 5) more than indicated in these plots.

After $700 \mathrm{~s}$ the platform is starting to gain a bit more amplitude in the pitch direction, but it does not become unstable. Control simulations of $2000 \mathrm{~s}$ are done to check this. The major consequence for introducing a failure to the closed-loop system is the non-symmetrical loading on the tower. This is especially prominent in the yaw direction, where the standard deviations are considerably larger than the values for the baseline controller (no fault occurs with baseline), while the mean values are lower. See the histogram in Figs. 9 and 10. The blade pitch movement for the LPV controller is shown in Fig. 8. It is seen that the three blades are more or less following each other until the fault occurs. At time equals $500 \mathrm{~s}$ the third blade pitch actuator stops moving. This is emulating a sensor failure. As the plot shows, only one pitch actuator is influenced by the fault.
Standard deviation for selected time series (normalized)

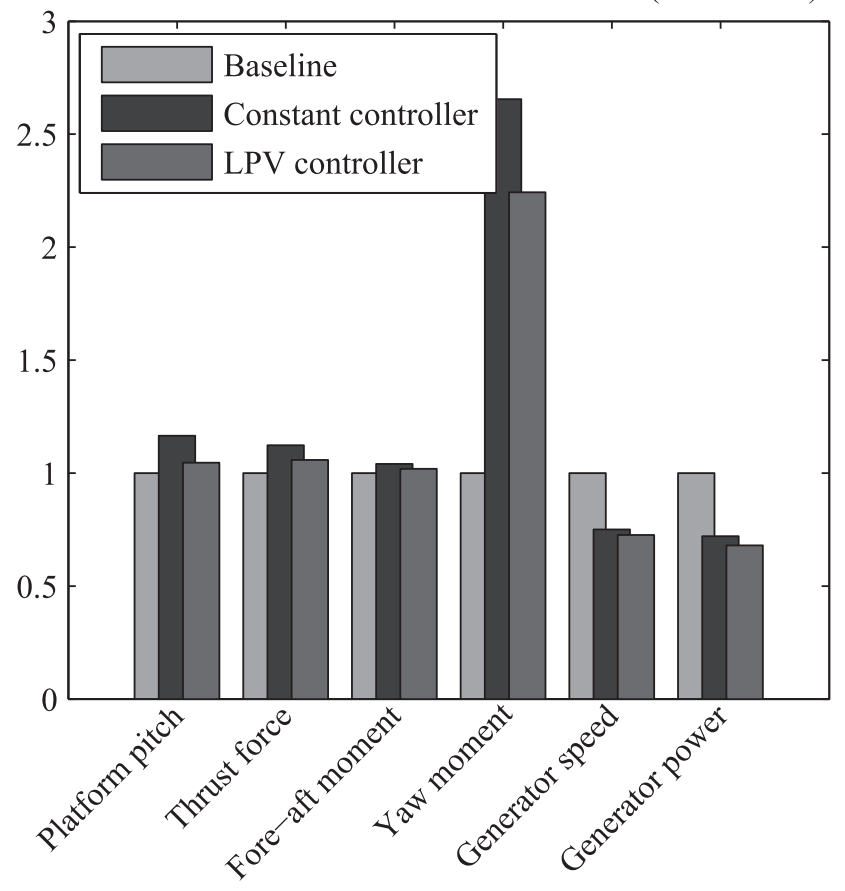

Fig.9 Normalised standard deviations for selected time series

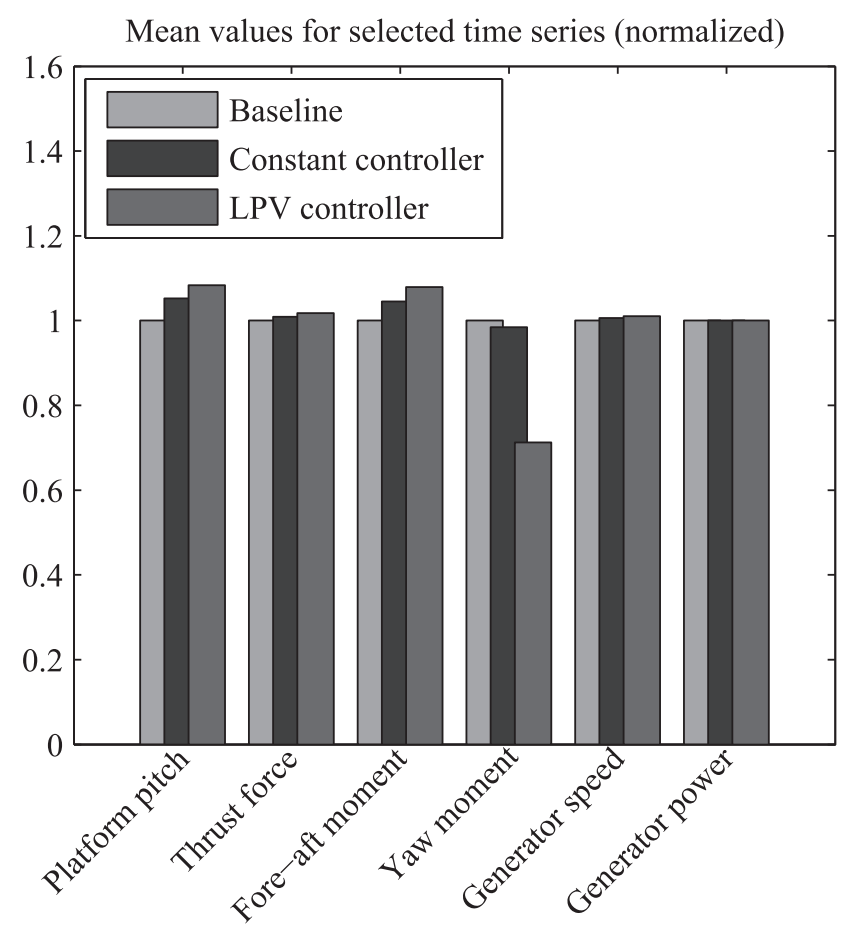

Fig.10 Normalised mean values for selected time series

From the histogram in Fig. 9, it is seen that the LPV controller has a lower standard deviation for generator speed and for generator power. The large yaw moment value is because of the fault which is introduced in the system. The constant controller also causes the system to experience a large standard deviation in the yaw moment, because of the fault. However, it has a better performance for the generator speed. The corresponding mean values can be seen in Fig. 10. 


\section{Conclusion and future work}

The purpose of this paper was to design an output-feedback LPV controller for an offshore wind turbine with constrained information. The scheduling parameter for the LPV controller is the effective wind speed. Based on available measurement an EKF is used to estimate the effective wind speed. The wind turbine model is obtained from the software FAST and all simulations are done in Matlab/Simulink. A wind turbine is a highly non-linear mechanism, and in order to use the controller design techniques proposed in this paper, a linearised model is needed. To linearise the model at only one operating point is a bit optimistic, therefore an LPV control approach is suggested. LPV control is a step in between linear control and non-linear control. The design is done on the basis of linear techniques and when it is implemented, the benefits from non-linear control are utilised. That is, it will perform and maintain stability in the whole operation region. With this method, LPV controller with constrained gain is constructed. Constrained gain means a special zero-non-zero pattern can be imposed on the gain matrix. In this paper, a controller gain with a diagonal structure is designed. From the simulation results it is seen that closed-loop system do behave according to the controller objectives and it maintains stability when a fault occurs to one of the sensors in the feedback loop.

Regarding the constrained gain matrix, this paper has focused on a diagonal structure. It is completely up to the designer to choose the zero-non-zero pattern for the gain matrix. In other cases different patterns than diagonal may be appropriate.

\section{Acknowledgment}

This work has been (partially) funded by Norwegian Centre for Offshore Wind Energy (NORCOWE) under grant 193821/S60 from Research Council of Norway (RCN). NORCOWE is a consortium with partners from industry and science, hosted by Christian Michelsen Research.

\section{References}

1 WWEA: 'World wide energy statistics half-year report 2012', no. 4, 2012

$24 \mathrm{C}$ offshore wind farms. [Online]. Available at http://www. 2.com/windfarms/

3 Skaare, B., Hanson, T.D., Nielsen, F.G., Yttervik, R., Hansen, A.M., Thomsen, K., Hansen T.J.: 'Integrated dynamic analysis of floating offshore wind turbines', Risø, Technical Report, 2007

4 Jonkman, J.: 'Definition of the floating system for phase IV of OC3', National Renewable Energy Laboratory', Technical Report NREL/TP500-47535, 2010.

5 Boyd, S., Ghaoui, L., Feron, E., Balakrishnan, V.: 'Linear matrix inequalities in systems and control theory', SIAM Stud. Appl. Math., (SIAM, Philadelphia, PA, Vol. 15, 1994)

6 Bianchi, F., Battista, H., Mantz, R.: 'Wind turbine control systems: principles, modelling and gain scheduling design' (Springer, 2007)

7 Østergaard, K., Stoustrup, J., Brath, P.: 'Linear parameter varying control of wind turbines covering both partial load and full load conditions', Int. J. Robust Nonlinear Control, 2009, 19, (1), pp. 92-116

8 Adegas, F.D., Stoustrup, J.: 'Structured control of LPV systems with application to wind turbines'. Am. Control Conf., 2012, pp. 756-761

9 Mohammadpour, J., Scherer, C.: 'Control of linear parameter varying systems with application' (Springer, 2012)

$10 \mathrm{Wu}$, L., Shi, P., Gao, H.: 'Delay-dependent robust $H_{\infty}$ and $L_{2}-L_{\infty}$ filtering for LPV systems with both discrete and distributed delays', IEE Proc.-Control Theory Appl., 2006, 153, (4), pp. 483-492
11 Wu, L., Lam, J., Wang, C.: 'Robust $H_{\infty}$ dynamic output feedback control of 2D linear parameter-varying systems', IMA J. Math. Control Inf., 2008, 26, (1), pp. 23-44

$12 \mathrm{Wu}, \mathrm{L}$., Yang, X., Li, F.: 'Nonfragile output tracking control of hypersonic air-breathing vehicles with an LPV model', IEEE/ASME Trans. Mechatronics, 2013, 18, (4), pp. 1280-1288

13 Bakka, T., Karimi, H.R.: 'Robust $H_{\infty}$ dynamic output feedback control synthesis with pole placement constraints for offshore wind turbine systems', Math. Prob. Eng., vol. 2012 (2012), Article ID 616507, 18 p.

14 Bakka, T., Karimi, H.R.: 'Gain scheduling for output $H_{\infty}$ control of offshore wind turbine'. Proc. 22nd Int. Offshore and Polar Engineering Conf., 2012

15 Zečević, A.I., Šiljak, D.D.: 'Design of robust static output feedback for large-scale systems', Trans. Autom. Control, 2004, 49, (11), pp. 2040-2044

16 Zečević, A.I., Šiljak, D.D.: 'Control design with arbitrary information structure constraints', Automatica, 2008, 44, (10), pp. 2642-2647

17 Zečević, A.I., Šiljak, D.D.: 'Control of complex systems: structural constraints and uncertainty' (Springer, 2010)

18 Rubió-Massegú, J., Rossell, J.M., Karimi, H.R., Palacios-Quiñonero, F.: 'Static output-feedback control under information structure constraints', Automatica, 2013, 49, (1), pp. 313-316

19 Sloth, C., Esbensen, T., Stoustrup, J.: 'Active and passive fault-tolerant LPV control of wind turbines'. Am. Control Conf., 2010, 2010, pp. 4640-4646

20 Sloth, C., Esbensen, T., Stoustrup, J.: 'Robust and fault-tolerant linear parameter-varying control of wind turbines', Mechatronics, 2011, 21, (4), pp. 645-659

21 Kamal, E., Aitouche, A., Ghorbani, R., Bayrat, M.: 'Robust fyzzy fault-tolerant control of wind energy conversion systems subjected to sensor faults', IEEE Trans. Sustain. Energy, 2012, 3, (2), pp. 231-241

22 Jonkman, J.: 'NWTC design codes' National Renewable Energy Center, 2010

23 Jonkman, J., Butterfield, S., Musial, W., Scott, G.: 'Definition of a 5-MW reference wind turbine for offshore system development', National Renewable Energy Center, Technical Report NREL/TP-50038060, 2009.

24 Knudsen, T., Bak, T., Soltani, M.: 'Prediction models for wind speed at turbine locations in a wind farm', Wind Energy, 2011, 14, (7), pp. 877-894

25 Jonkman, B.: 'Turbsim users guide: Version 1.50', National Renewable Energy Center, Technical Report, 2009.

\section{Appendix}

Blade pitch actuator model

$$
\begin{aligned}
\dot{x}_{\mathrm{p}} & =A_{\mathrm{p}} x_{\mathrm{p}}+B_{\mathrm{p}} u \\
y & =C_{\mathrm{p}} x_{\mathrm{p}}
\end{aligned}
$$

where

$$
A_{\mathrm{p}}=\left[\begin{array}{cc}
-2 \omega_{n} \zeta & -\omega_{n}^{2} \\
1 & 0
\end{array}\right], \quad B_{\mathrm{p}}=\left[\begin{array}{l}
1 \\
0
\end{array}\right], \quad C_{\mathrm{p}}=\left[\begin{array}{ll}
0 & \omega_{\mathrm{n}}^{2}
\end{array}\right]
$$

The natural frequency is $\omega_{\mathrm{n}}=0.88$ and the damping ratio is $\zeta=0.9$. There is a total of three of these pitch actuators models in the turbine model, one for each blade.

Constant constrained gain: $K_{\text {constant }}=\operatorname{diag}\{0.0315,0.0008$, $0.00017\}$

Lyapunov matrices used online to calculate the LPV constrained gain

$$
\begin{aligned}
Y_{r 0} & =\operatorname{diag}\{0.0006,-0.3788,0.0005\} \times 10^{-3} \\
Y_{r 1} & =\operatorname{diag}\{0.0008,1.2192,-0.0002\} \times 10^{-2} \\
X_{r 0} & =\operatorname{diag}\left\{1.9527 \times 10^{-4}, 13.9305,0.0012\right\} \\
X_{r !} & =\operatorname{diag}\left\{7.7424 \times 10^{-8}, 0.0231,1.5532 \times 10^{-6}\right\}
\end{aligned}
$$

